\title{
Avaliação de diferentes procedimentos de acabamento e polimento sobre a rugosidade de superfície de resina composta submetida à escovação com dentifrício
}

\author{
Evaluation of different finishing and polishing procedures on surface \\ roughness of composite resin submitted to brush with dentifrice \\ Evaluación de diferentes procedimientos de acabado y pulido \\ sobre la rugosidad de superficie de resina compuesta \\ sometida a la acción del cepillado con dentífrico \\ Bruna de Oliveira REIS ${ }^{1}$ \\ Fabiana Ferres BROGIN ${ }^{2}$, \\ Anderson CATELAN ${ }^{3}$, \\ André Luiz Fraga BRISO ${ }^{1}$ \\ Paulo Henrique DOS SANTÓ ${ }^{2}$ \\ ${ }^{I}$ Departamento de Odontologia Restauradora, Faculdade de Odontologia, UNESP Univ. Estadual Paulista, \\ 16015-050 Araçatuba - SP, Brasil \\ ${ }^{2}$ Departamento de Materiais Odontológicos e Prótese, Faculdade de Odontologia, UNESP Univ. Estadual Paulista, \\ 16015-050 Araçatuba-SP, Brasil \\ ${ }^{3}$ Departamento de Odontologia, Faculdade de Odontologia, UNITAU Univ. de Taubaté, \\ 12020-200 Taubaté-SP, Brasil
}

\section{Resumo}

Introdução: As resinas compostas são os materiais restauradores que mais evoluíram na Odontologia Estética. Em condições normais, o desgaste dos materiais é proveniente da mastigação e do atrito dos abrasivos contidos nos cremes dentais durante a escovação. Este processo abrasivo pode também comprometer o acabamento do compósito, aumentando também a sua rugosidade. Objetivo: Avaliar os efeitos de diferentes procedimentos de acabamento e polimento sobre a rugosidade de superfície de diferentes resinas compostas, submetidas a ciclos de escovação mecânica. Materiais e Métodos: Quarenta corpos-de-prova foram preparados para cada resina composta (Filtek Supreme XT-3M/Espe e Esthet-X - Dentsply) e submetidos ao polimento (Sof-Lex - 3M/Espe, PoGo - Dentsply e Biscover LV Bisco e controle). Para a análise inicial da rugosidade de superfície foi empregado rugosímetro SJ 401 (Mitutoyo). Após a leitura inicial, os corpos-de-prova foram submetidos a 30000 ciclos de escovação utilizando o dentifrício Colgate Máxima Proteção Anticárie (ColgatePalmolive), seguida de nova leitura da rugosidade. Amostras representativas de cada grupo foram metalizadas e levadas à microscopia eletrônica de varredura. Resultados: Houve diferença estatisticamente significante de rugosidade $(\mathrm{p}<0,05)$ tanto entre as resinas compostas quanto entre as técnicas de acabamento e polimento. A resina Esthet-X apresentou os maiores valores iniciais de rugosidade, sendo que após a escovação não houve diferença com a Filtek Supreme XT. Conclusão: dentre as técnicas de acabamento e polimento, o selamento com Biscover LV possibilitou os menores valores de rugosidade de superfície para ambas as resinas.

Descritores: Propriedades de Superfície; Resinas Compostas; Escovação Dentária.

\section{Abstract}

Introduction: Composite resins are the restorative materials that have most evolved in Aesthetic Dentistry. Under normal conditions, the wear of the materials comes from mastication and friction of the abrasives contained in the toothpastes during brushing. This abrasive process can also compromise the finish of the composite, also increasing its roughness. Objetives: To evaluate the effect of different finishing and polishing systems on the surface roughness of resin composites, submitted to mechanical brushing. Materials and Methods: Forty samples of each composite ((Filtek Supreme XT-3M/Espe and Esthet-X - Dentsply) were made and submitted to the following finishing and polishing systems: Sof-Lex - 3M/Espe, PoGo - Dentsply, Biscover LV - Bisco and control. The initial measurements of surface roughness was made on the SJ 401 perfilometer (Mitutoyo). After the initial measumentes, the samples were submitted to 30,000 cycles of mechanical brushing using a Colgate Máxima Proteção Anticárie (Colgate-Palmolive) dentifrice, followed by the final measurements of surface roughness. Representative samples of each group were gold sputtered and analyzed by scanning eletron microscope. Results: There was a statistically significant difference of roughness $(p<0.05)$ both as composite resins and between finishing and polishing techniques. The Esthet-X resin had the highest initial values of, and after the brushing there was no difference with a Filtek Supreme XT. Conclusion: Among the techniques of finishing and polishing, the sealing with Biscover LV allowed the smallest values of surface roughness for both resins.

Descriptors: Surface Properties, Composite Resins, Toothbrushing.

\section{Resumen}

Introducción: Las resinas compuestas son los materiales restauradores que más evolucionan en la Odontología Estética. En condiciones normales, el desgaste de los materiales procede de la masticación y de la fricción de los abrasivos contenidos en las cremas dentales durante el cepillado. Este proceso abrasivo puede también comprometer el acabado del compuesto, aumentando también su rugosidad. Objetivo: Evaluar los efectos de diferentes procedimientos de acabado y pulido sobre la rugosidad superficial de diferentes resinas compuestas, sometidas a ciclos de cepillado mecánico. Materiales y Métodos: Cuarenta cuerpos de prueba se prepararon para cada resina compuesta (Filtek Supreme XT-3M / Espe y Esthet-X - Dentsply) y sometidos al pulido (Sof-Lex - 3M / Espe, PoGo - Dentsply y Biscover LV - Bisco y control). Para el análisis inicial de la rugosidad superficial se empleó el rugosímetro SJ 401 (Mitutoyo). Después de la lectura inicial, los cuerpos de prueba fueron sometidos a 30000 ciclos de cepillado utilizando el dentifrício Colgate Máxima Protección Anticaria (ColgatePalmolive), seguida de nueva lectura de la rugosidad. Las muestras representativas de cada grupo se metalizaron y se llevaron a la microscopía electrónica de exploración. Resultados: Hubo diferencia estadísticamente significativa de rugosidad $(p<0,05)$ tanto entre las resinas compuestas como entre las técnicas de acabado y pulido. La resina Esthet-X presentó los mayores valores iniciales de rugosidad, siendo que después del cepillado no hubo diferencia con la Filtek Supreme XT. Conclusión: entre las técnicas de acabado y pulido, el sellado con Biscover LV posibilitó los menores valores de rugosidad de superficie para ambas resinas.

Descriptores: Propiedades de superficie; Resinas Compuestas; Cepillado Dental.

\section{INTRODUÇÃO}

As resinas compostas são os materiais restauradores que mais evoluíram no campo da Odontologia Estética, sendo constituídas de componentes orgânicos e inorgânicos que interferem em suas características e propriedades de manipulação. Uma das propriedades físicas que o material restaurador deve exibir é a capacidade de resistir ao desgaste. Em condições normais, o desgaste dos materiais é proveniente da mastigação e do atrito dos abrasivos contidos nos cremes dentais durante a escovação ${ }^{1,2}$. Este processo abrasivo pode também comprometer o acabamento do compósito, aumentando também a sua rugosidade $^{3}$. 
O avanço tecnológico dos materiais tem propiciado o desenvolvimento de compósitos restauradores nanoparticulados os quais mostraram possuir propriedades físicas relevantes, resistência ao desgaste e também melhorias no acabamento e polimento superficial. Além do mais, as propriedades estéticas dos nanoparticulados mostraram permitir seu uso clínico em restaurações anteriores e posteriores ${ }^{4,5}$

Vale destacar que um bom acabamento e polimento dos compósitos são passos importantes na obtenção da estética e longevidade das restaurações, diminuindo o acúmulo de placa, a irritação gengival e a alteração de cor da restauração ${ }^{6,7}$

Pequenos defeitos são comumente encontrados na superfície das restaurações, particularmente em áreas de grande tensão. Esses defeitos podem ser criados pelos procedimentos de acabamento e polimento, aumentando o desgaste e rugosidade das restaurações ${ }^{8}$. Segundo Bollen et al. ${ }^{9}$ existe um limiar de rugosidade de superfície $(\mathrm{Ra}=0,2 \mu \mathrm{m})$, acima do qual favorece o acúmulo da placa bacteriana.

Em 1989, Ratanapridakul et al. ${ }^{10}$ sugeriram a aplicação de selantes na superfície dos materiais resinosos visando o preenchimento das microrrachaduras e o reforço da matriz orgânica, aumentando a resistência ao desgaste das resinas e melhorando a integridade marginal ${ }^{11}$.

Clinicamente há alternativas para realizar acabamento e polimento das restaurações. As formas mais populares são as pontas diamantadas, taças de borracha e pastas abrasivas, sendo primordial aplicar progressivamente as granulações ${ }^{12}$. Mais recentemente foi introduzido no mercado o Biscover, uma resina fotopolimerizável usada como selador superficial, que poderia eliminar a necessidade do polimento manual e consequentemente seus efeitos na rugosidade superficial.

Independente da composição, os compósitos odontológicos sofrem tipos similares de alterações microestruturais, ocorridas principalmente na parte superficial, tais alterações tidas como microdefeitos ou microfendas são avaliadas por meio da microscopia eletrônica de varredura, sendo este dispositivo uma forma útil de qualificar os materiais usados para polimento, dando maior confiabilidade aos trabalhos realizados ${ }^{13}$.

Neste sentido, faz-se necessário estudar os efeitos de diferentes procedimentos de acabamento e polimento sobre a rugosidade de superfície desses novos materiais introduzidos no mercado e a efetividade do selante de superfície frente ao processo abrasivo da escovação. O objetivo do presente estudo foi avaliar e comparar o efeito de três sistemas de acabamento e polimento sobre a rugosidade superficial de dois tipos de resina composta e analisar alterações topográficas por meio da microscopia eletrônica de varredura.

\section{MATERIAL E MÉTODO}

Quarenta corpos-de-prova dos compósitos Filtek Supreme XT (3M/ESPE) e Esthet-X (Dentsply) foram confeccionados utilizando matriz metálica rosqueável de $2 \mathrm{~mm}$ de profundidade e $4 \mathrm{~mm}$ de diâmetro. Os materiais foram inseridos na matriz metálica utilizando uma espátula Thompson, preenchendo completamente a matriz. Sobre o compósito foi posicionada uma tira de poliéster e uma lâmina de vidro, a fim de nivelar o material na borda superior da matriz e eliminar o excesso do mesmo. $\mathrm{O}$ compósito foi fotoativado com equipamento Ultraled (Dabi Atlante, Ribeirão Preto, SP, Brasil) na potência máxima do aparelho $\left(700 \mathrm{~mW} / \mathrm{cm}^{2}\right)$ pelo tempo de 20 segundos, seguindo as recomendações dos fabricantes. As dimensões dos corpos de prova foram aferidas com auxílio de paquímetro digital (Mitutoyo America Corporation, Suzano, SP, Brasil) e na seqüência foram armazenados em água destilada a $37^{\circ} \mathrm{C}$ (ECB-2, Adamo Produtos para Laboratórios Ltda, Ribeirão Preto, SP, Brasil).

Após 24 horas, os corpos-de-prova foram divididos em quatro grupos $(n=10)$ de acordo com o procedimento de acabamento e polimento utilizado:

○ Grupo I - Sistema Sof-Lex (3M/ESPE, St Paul, MN, USA), aplicando-se os discos de granulação média, fina e superfina, durante 30 segundos cada.

○ Grupo II - Sistema PoGo (Dentsply Latin America, Petrópolis, RJ, Brasil), aplicando-se o disco microdiamantado durante 30 segundos.

- Grupo III - Resina líquida Biscover LV (Bisco Schaumburg, IL, USA), fotoativando-a durante 30 segundos. Previamente à aplicação da resina líquida, as amostras foram condicionadas com ácido fosfórico 32\% (Uni-Etch, Bisco Schaumburg, IL, USA) durante 15 segundos, seguido de lavagem e secagem com jatos de ar, de acordo com as recomendações do fabricante.

- Grupo IV - Controle, sem a realização de acabamento ou polimento.

Para análise da rugosidade de superfície, os corposde-prova foram levados individualmente ao rugosímetro portátil SJ-401 (Mitutoyo America Corporation, Suzano, SP, Brasil) e as leituras de rugosidade analisadas no programa Surfpak (Mitutoyo America Corporation, Suzano, SP, Brasil). O padrão de rugosidade utilizado foi o Ra, o qual representa a média aritmética entre picos e vales registrados. Foi utilizado um cut-off de $0,25 \mathrm{~mm}$, necessário para maximizar a filtragem da ondulação superficial, sendo efetuadas quatro leituras em diferentes posições, sempre com a agulha passando pelo centro geométrico da amostra. Uma pequena marca na lateral do corpo-de-prova foi feita para orientação da segunda leitura da rugosidade.

Após as leituras da rugosidade de superfície, os corpos-de-prova foram submetidos ao ensaio mecânico de escovação, utilizando a ponta ativa de escovas dentais OralB 35 (Gilette do Brasil) com cerdas de secção hexagonal extra-macias. A ponta ativa da escova foi fixada na máquina de escovação MSet (Marcelo Nucci ME, São Carlos, SP, Brasil), com capacidade de escovar dez corpos-de-prova simultaneamente. A amplitude de movimento horizontal das escovas dentais foi de $40 \mathrm{~mm}$.

O slurry composto de 6 gramas do dentifrício Colgate Máxima Proteção Anticaries (Colgate-Palmolive, Osasco, SP, Brasil) misturado a $10 \mathrm{ml}$ de saliva artificial (Dermavena) foi preparado e todos os corpos-de-prova foram submetidos a movimentos lineares de escovação, totalizando 30000 ciclos, com freqüência de 7 movimentos por segundo, sob carga de 300gf, para simular a força empregada durante os procedimentos de higiene oral. Foram utilizadas uma escova dental e uma solução contendo dentifrício para escovação de cada corpo-de-prova, sendo que as mesmas foram trocadas a cada troca de corpo-deprova. A cada 2000 ciclos de escovação, a solução contendo dentifrício foi homogeneizada novamente, para evitar a deposição de partículas. Após o ensaio de escovação, todos os corpos-de-prova foram avaliados novamente quanto à rugosidade de superfície, seguindo a orientação das marcações realizadas na lateral do corpo-de-prova.

Amostras representativas de cada grupo experimental foram limpas em ultra-som Cristófoli (Equipamentos de 
Biossegurança Ltda, Campo Mourão, PR, Brasil), metalizadas com ouro (SCD 050 - Balzers) e levadas à microscopia eletrônica de varredura (Jeol modelo JSM 5600 LV) para análise qualitativa das superfícies dos materiais. Para análise das superfícies nos grupos experimentais, novas amostras foram confeccionadas, polidas e lidas sem a realização da escovação simulada.

Os dados obtidos foram submetidos à Analise de Variância e as médias comparadas pelo teste de Tukey $(\alpha=0,05)$. Foi verificada a variável rugosidade de superfície, sendo que existiram três fatores de variação (resina composta, tipo de sistema de acabamento e polimento e os períodos de avaliação antes e após a escovação mecânica).

\section{RESULTADOS}

Os resultados mostraram que independente da técnica de acabamento e polimento, a resina Filtek Supreme XT apresentou menor média inicial de rugosidade $(0,087 \pm 0,021 \mu \mathrm{m})$ que a resina Esthet $X(0,108 \pm 0,023 \mu \mathrm{m})$ $(\mathrm{p}<0,05)$. Após a escovação mecânica, não houve diferença significante entre as resinas (Figura 1).

A Figura 2 ilustra que para a resina Esthet $\mathrm{X}$, os menores valores de rugosidade de superfície antes da escovação foram obtidos para o grupo Biscover LV $(0,084$ $\pm 0,047 \mu \mathrm{m})$, sem diferença estatística para o grupo controle $(0,089 \pm 0,033 \mu \mathrm{m})$ e Sof-Lex $(0,103 \pm 0,011 \mu \mathrm{m})(\mathrm{p}>0,05)$. $\mathrm{O}$ grupo polido com o sistema PoGo apresentou maior média de rugosidade de superfície $(0,124 \pm 0,043 \mu \mathrm{m})$, sem diferença estatística para o grupo Sof-Lex. Após a escovação, o grupo Biscover LV apresentou a menor média de rugosidade de superfície $(0,087 \pm 0,020 \mu \mathrm{m})$, com diferença estatística para os demais grupos $(p<0,05)$. Para a resina Filtek Supreme XT, não houve diferença estatística entre todas as técnicas de acabamento e polimento antes da escovação mecânica ( $p>0,05)$. Após a escovação, o menor valor de rugosidade de superfície foi obtido para o Biscover LV $(0,076 \pm 0,006 \mu \mathrm{m})$, com diferença estatística para os demais grupos $(\mathrm{p}<0,05)$ (Figura 3$)$.

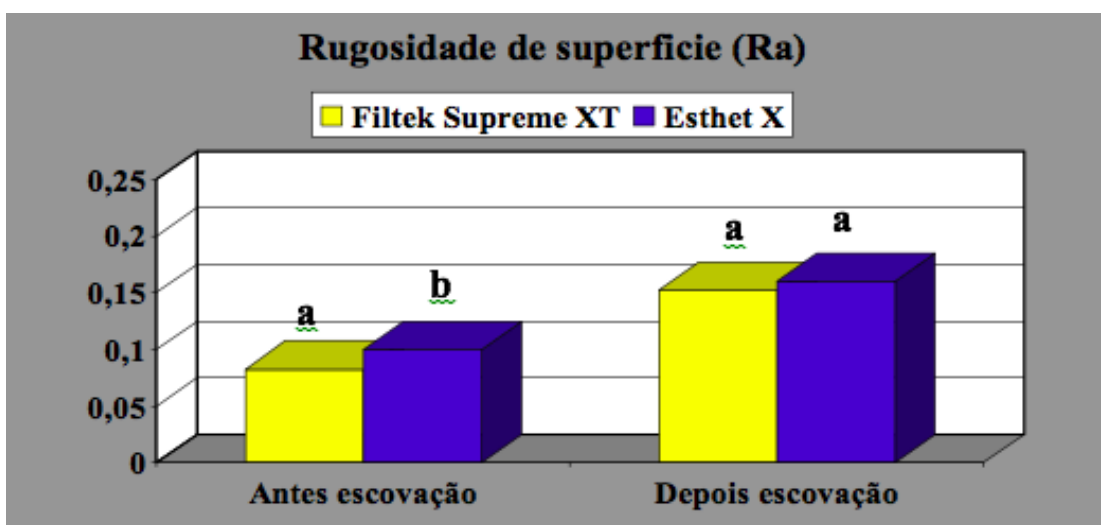

Figura 1: Comparação dos valores de rugosidade de superfície para as resinas compostas antes e depois da escovação, independente da técnica de acabamento e polimento.

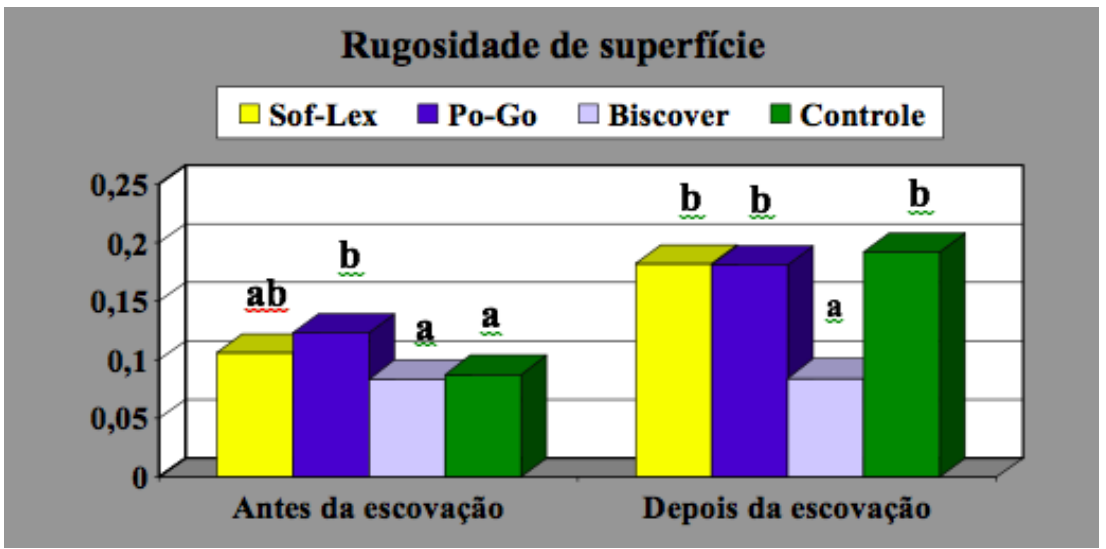

Figura 2: Comparação dos valores de rugosidade de superfície entre as técnicas de acabamento e polimento para a resina Esthet X, antes e após a escovação mecânica.

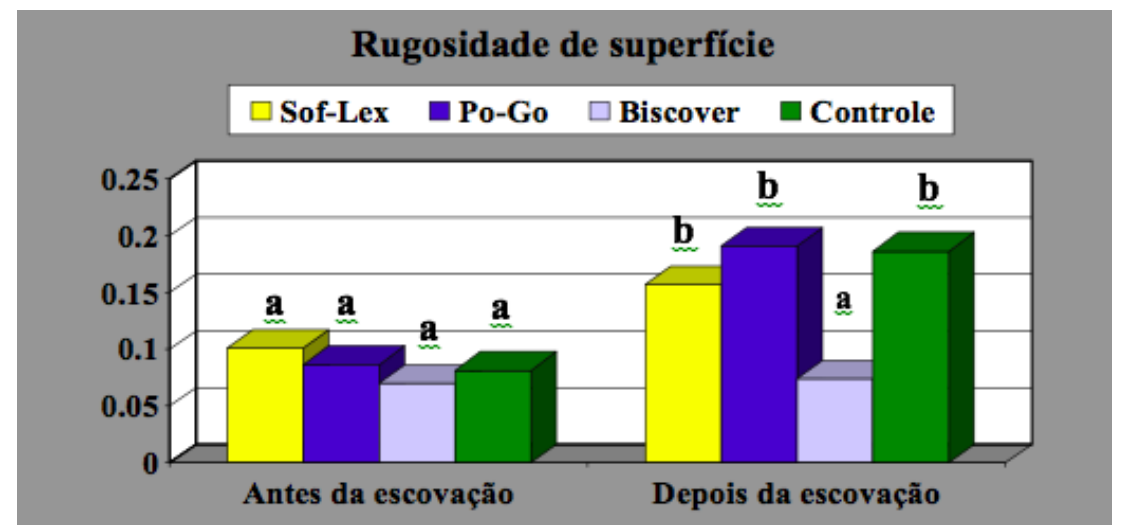

Figura 3: Comparação dos valores de rugosidade de superfície entre as técnicas de acabamento e polimento para a resina Filtek Supreme XT, antes e após a escovação mecânica.

Para a resina Esthet X (Figura 4), houve diferença significante nos valores de rugosidade de superfície antes e após a escovação para todas as técnicas de acabamento e polimento, à exceção do Biscover $\mathrm{LV}$, cujo valor antes da escovação $(0,084 \pm 0,047 \mu \mathrm{m})$, não diferiu estatisticamente do valor de rugosidade após a escovação $(0,087 \pm$ $0,020 \mu \mathrm{m})$. O mesmo pode-se observar para a resina Filtek Supreme XT (Figura 5), cujo valor de rugosidade antes da escovação para o grupo Biscover LV $(0,074 \pm 0,010 \mu \mathrm{m})$ foi semelhante ao obtido após a escovação $(0,076 \pm 0,006 \mu \mathrm{m})$.

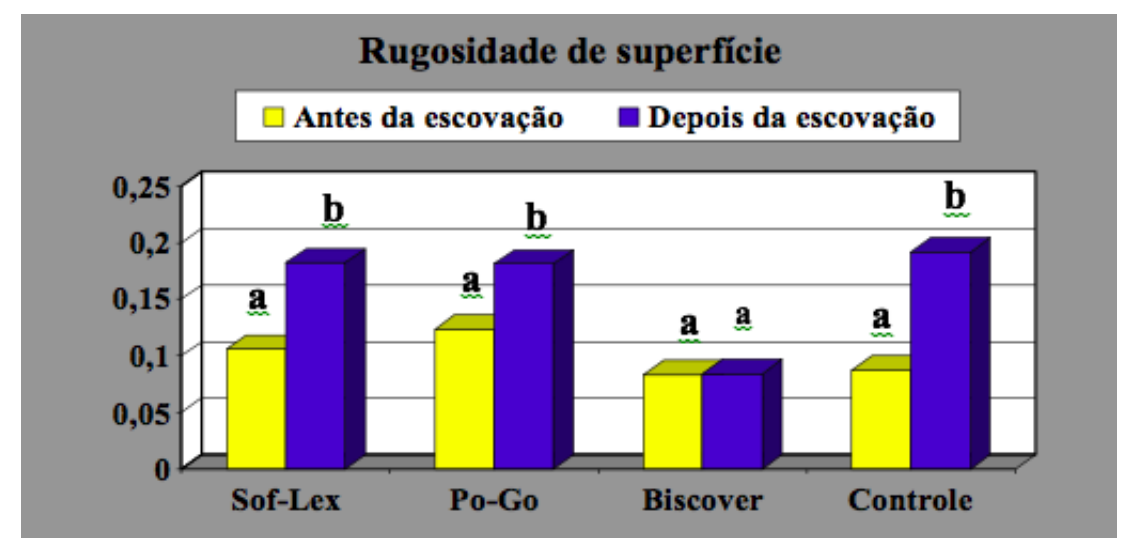

Figura 4: Comparação dos valores de rugosidade de superfície, antes e depois da escovação para a resina Esthet $\mathrm{X}$, de acordo com as técnicas de acabamento e polimento.

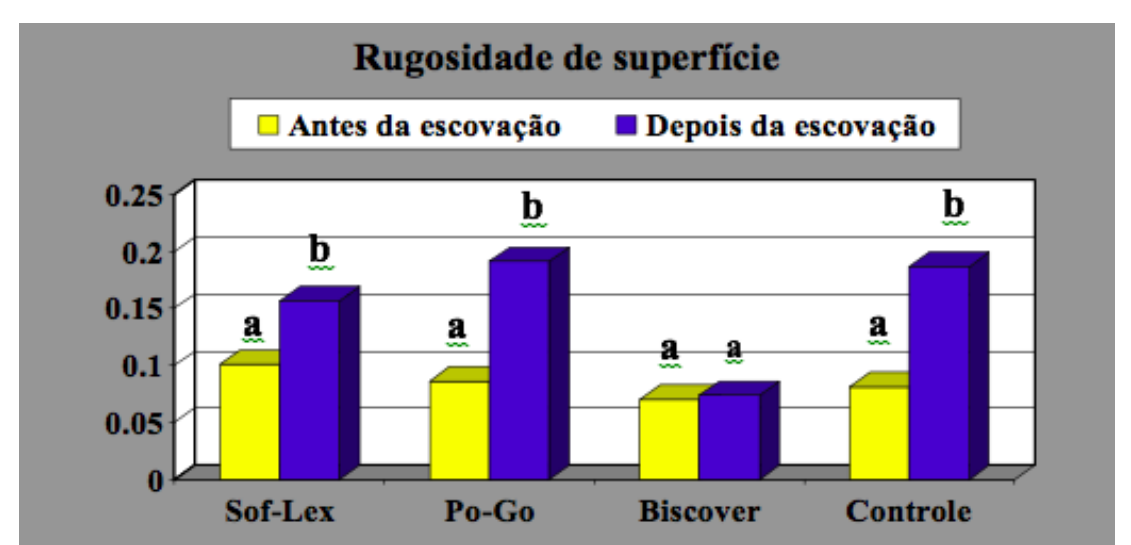

Figura 5: Comparação dos valores de rugosidade de superfície, antes e depois da escovação para a resina Supreme XT, de acordo com as técnicas de acabamento e polimento.

A Figura 6 ilustra, por meio da microscopia eletrônica de varredura, a característica superficial da resina composta Esthet $\mathrm{X}$ nos quatro grupos experimentais, de acordo com o acabamento e polimento realizado antes do processo de escovação mecânica.

A Figura 7 ilustra a mesma resina com os mesmos grupamentos experimentais após o ensaio mecânico de escovação. A Figura 8 ilustra, por meio da microscopia eletrônica de varredura, a característica superficial da resina composta Supreme XT nos quatro grupos experimentais, de acordo com o acabamento e polimento realizado antes do processo de escovação mecânica. A Figura 9 ilustra a mesma resina com os mesmos grupamentos experimentais após o ensaio mecânico de escovação. 


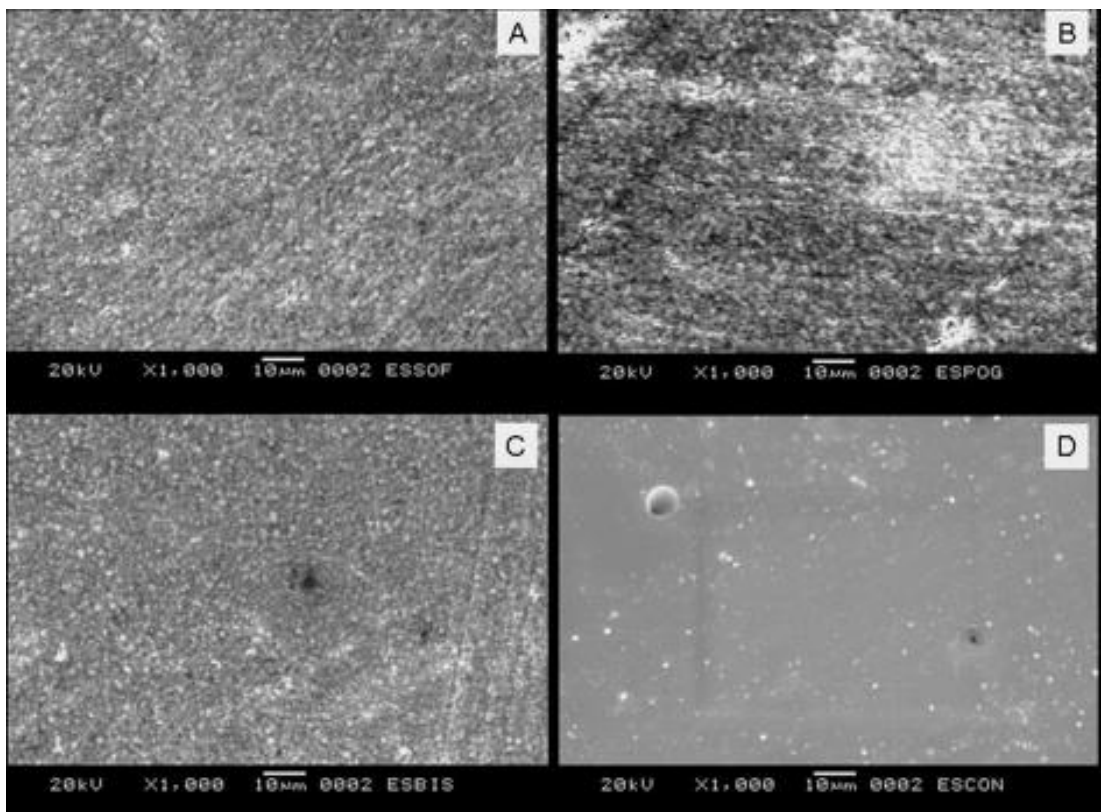

Figura 6: Resina Esthet-X antes da escovação mecânica (A - Sof-Lex; B - Po-Go; C - Biscover LV e D - controle)

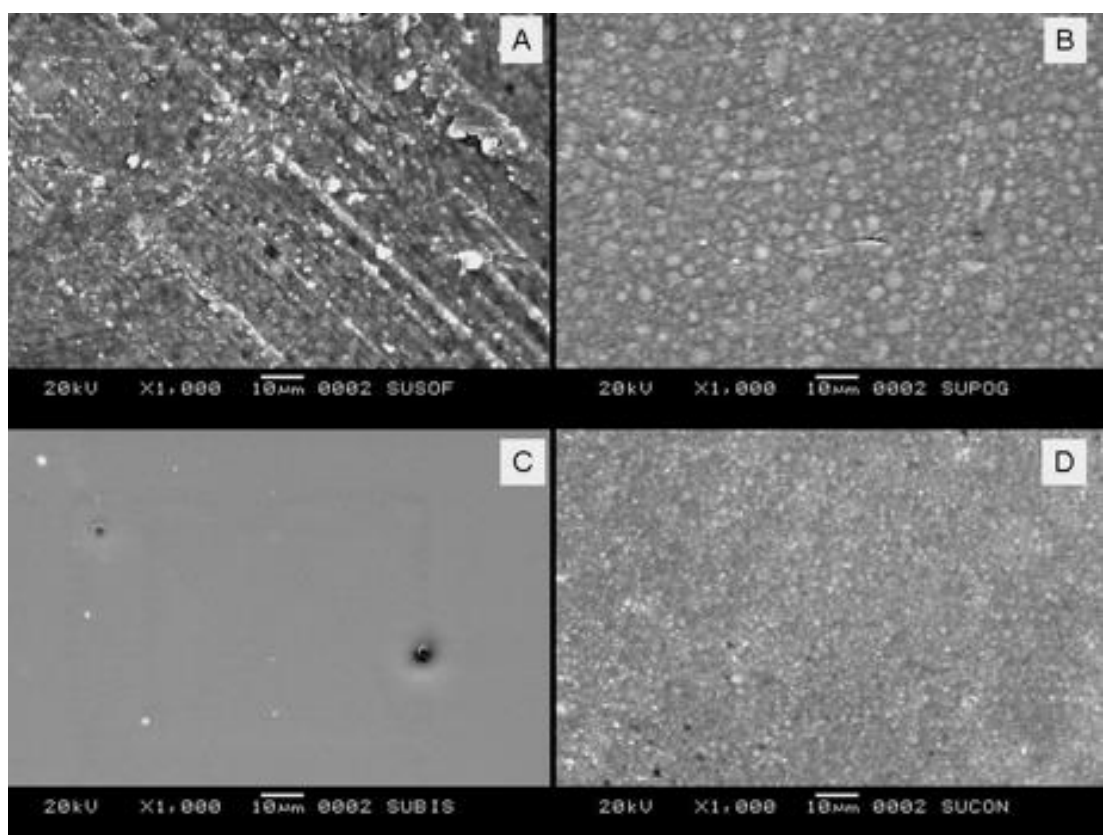

Figura 8: Resina Supreme XT antes da escovação (A - Sof-Lex; B - PoGo; C - Biscover LV e D - controle).

\section{DISCUSSÃO}

O objetivo da excelência nos procedimentos de acabamento e polimento é melhorar a estética e a longevidade das restaurações de resina compostas ${ }^{14}$. No entanto, em virtude da diferença nos valores de dureza entre a matriz orgânica e as partículas inorgânicas, estes procedimentos podem não desgastar uniformemente a superfície das resinas compostas ${ }^{15-17}$. De acordo com Sarac et al. ${ }^{17}$, um bom sistema de acabamento para resina composta deve possuir partículas mais duras que as partículas de carga dos materiais, caso contrário, o agente de polimento removerá somente a matriz orgânica, expondo as partículas de carga na superfície do material. A lisura de materiais heterogêneos, como as resinas compostas, também é influenciada pela estrutura interna do material, como tamanho e distribuição das partículas de carga ${ }^{18} \mathrm{e}$, por estes motivos, fabricantes têm reduzido o tamanho das partículas, na tentativa de otimizar o acabamento e polimento das restaurações ${ }^{19}$.

Os resultados deste estudo mostram que, independente da técnica de acabamento e polimento, a resina nanoparticulada Filtek Supreme XT apresentou menor média inicial de rugosidade de superfície. De acordo com Nagem-Filho et al. $^{6}$ e Yap et al. ${ }^{5}$, diferenças significantes nos valores de Ra poderiam ser atribuídas ao tamanho das partículas de carga e sua habilidade em formar uma camada polimérica homogênea quando o material é polimerizado sob pressão. Segundo Joniot et al. ${ }^{20}$, a

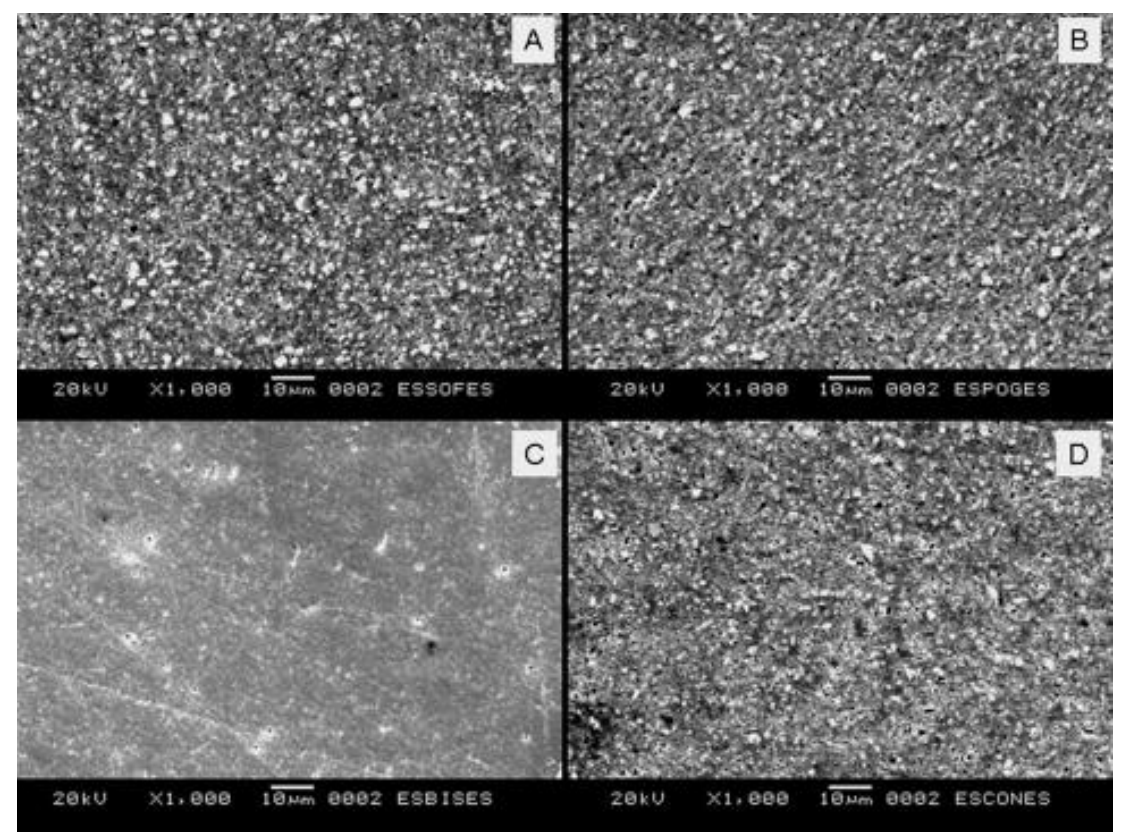

Figura 7: Resina Esthet-X após a escovação mecânica (A - Sof-Lex; B Po-Go; C - Biscover LV e D - controle).

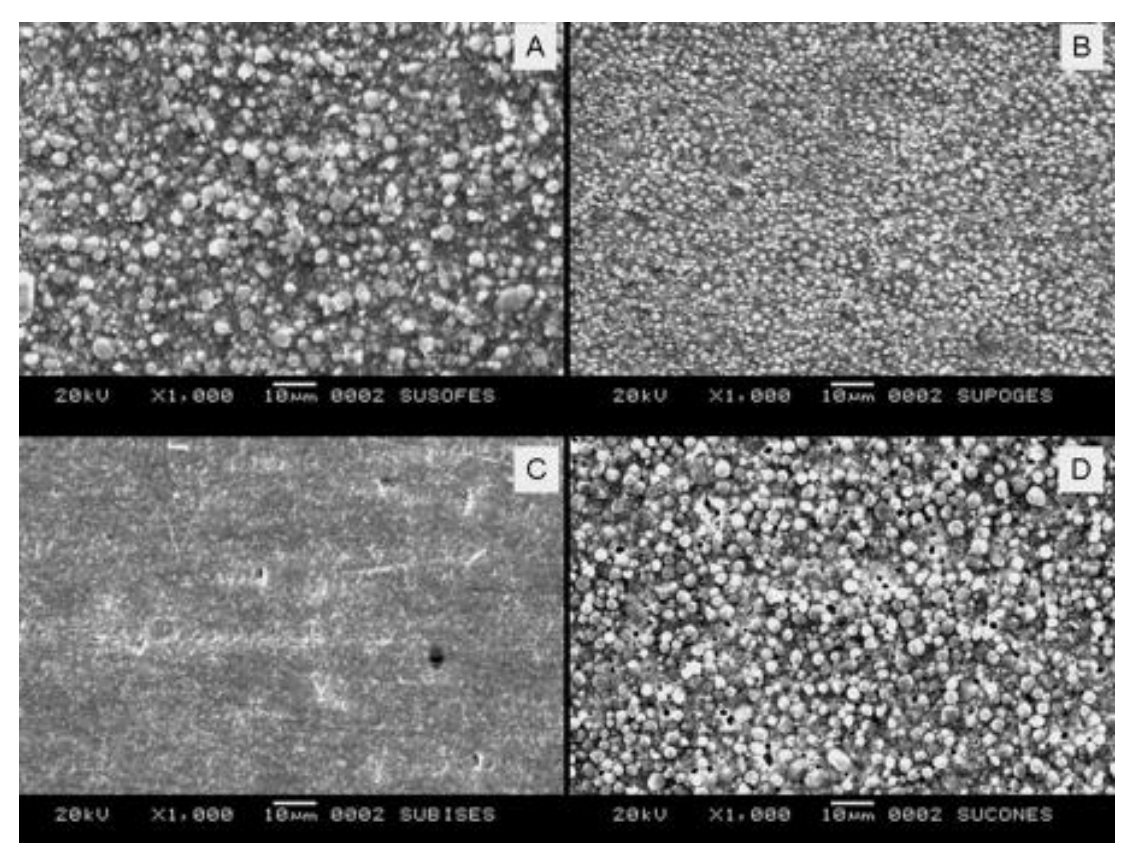

Figura 9: Resina Supreme XT após a escovação (A - Sof-Lex; B - PoGo; C - Biscover LV e D - controle).

qualidade final da superfície polida depende da forma e do tamanho das partículas dos materiais, sua proporção e composição. A resina Filtek Supreme XT apresenta, em sua composição, nanopartículas com tamanho médio de 5-20nm $\mathrm{nm}^{4}$ enquanto que a resina microhíbrida Esthet $\mathrm{X}$, possui tamanho médio das partículas de $1 \mu \mathrm{m}$, ou seja, o material com partículas maiores obteve a superfície mais rugosa $^{19}$. Além disso, a resina Esthet $X$ possui maior porcentagem de massa/volume, o que também contribui para uma superfície mais rugosa ${ }^{21}$.

Os resultados deste estudo mostram que, independente da técnica de acabamento e polimento, a resina nanoparticulada Filtek Supreme XT apresentou menor média inicial de rugosidade de superfície. De acordo com Nagem-Filho et al. $^{6}$ e Yap et al. ${ }^{5}$, diferenças significantes nos valores de Ra poderiam ser atribuídas ao tamanho das partículas de carga e sua habilidade em formar uma camada polimérica homogênea quando o material é polimerizado sob pressão. Segundo Joniot et al. ${ }^{20}$, a qualidade final da superfície polida depende da forma e do tamanho das partículas dos materiais, sua proporção e composição. A resina Filtek Supreme XT apresenta, em sua composição, nanopartículas com tamanho médio de 5$20 \mathrm{~nm}^{4}$, enquanto que a resina microhíbrida Esthet X, possui tamanho médio das partículas de $1 \mu \mathrm{m}$, ou seja, o material com partículas maiores obteve a superfície mais rugosa ${ }^{20}$. Além disso, a resina Esthet $\mathrm{X}$ possui maior porcentagem de 
massa/volume, o que também contribui para uma superfície mais rugosa ${ }^{21}$.

Pela microscopia eletrônica de varredura pode-se observar também que a resina Supreme XT apresenta uniformidade na distribuição de partículas de carga (Figuras 8 e 9). A maior uniformidade na distribuição das partículas poderia também ter contribuído para os menores valores de rugosidade encontrados para a resina Supreme $\mathrm{XT}^{4}$. Além disso, de uma maneira geral, as técnicas de acabamento e polimento da resina Esthet $\mathrm{X}$ deixaram a resina com riscos superficiais mais nítidos (Figura 6), comparativamente à resina Supreme XT (Figura 8), à exceção do grupo polido com Sof-Lex (Figura 6A).

Após a escovação mecânica, houve aumento nos valores de rugosidade de superfície para as duas resinas Filtek Supreme XT e Esthet-X sem diferenças significantes entre elas (Figura 1). O aumento da rugosidade após a escovação se deve ao atrito das partículas abrasivas contidas nos dentifrícios ${ }^{22}$ com os materiais. A sílica, o carbonato de cálcio, o fosfato de cálcio, o óxido de alumínio e a pedra pomes abrasionam a superfície das restaurações causando o aparecimento de pequenos riscos, removendo camadas mais superficiais da matriz orgânica, expondo partículas de carga mais duras e, conseqüentemente, aumentando a rugosidade de superfície ${ }^{1,3,23}$. As imagens em microscopia eletrônica de varredura após escovação demonstram a maior exposição das partículas de carga após o ensaio mecânico de escovação, o que provavelmente tenha contribuído para o aumento nos valores de rugosidade (Figuras 7 e 9).

A Figura 2 mostra que os menores valores de rugosidade $(\mathrm{Ra})$ obtidos antes da escovação foram obtidos pelos grupos Biscover LV, controle e polido com Sof-Lex, enquanto após a escovação, o grupo Biscover LV diferenciou-se estatisticamente dos demais grupos, apresentando os menores valores de rugosidade de superfície (Figura 2). Esses resultados concordam com os dados de Sarac et al. ${ }^{17}$, que verificaram que o selante Biscover propicia menores valores de rugosidade de superfície quando comparados com os discos de polimento. Segundo os autores, o material selador preenche os microdefeitos estruturais formados durante a técnica de acabamento e polimento, fornecendo uma superfície mais uniforme, regular e com pouca rugosidade.

Vale lembrar que grupo controle e o polido com o sistema Sof-Lex não apresentaram diferenças significativas para o grupo Biscover LV antes da escovação. No entanto, segundo Ozgunaltay et al. ${ }^{15}$ e Gedik et al. ${ }^{16}$, a tira de poliéster utilizada no grupo controle, forma uma superfície lisa e uniforme no compósito restaurador e que, embora fosse um instrumento versátil, apresentaria uma dificuldade clínica em dar contorno e forma às restaurações. Assim como o grupo controle, o grupo Sof-Lex também demonstrou habilidade em produzir uma superfície lisa e uniforme, devido a sua capacidade de cortar/desgastar as partículas de carga e a matriz resinosa igualmente, não provocando deslocamento das partículas ${ }^{15,24}$. A imagem $6 \mathrm{~A}$ ilustra o aspecto da resina Esthet $\mathrm{X}$ após o polimento com os discos Sof-Lex, não demonstrando elevação das partículas de carga na superfície do compósito, embora com aspecto mais riscado que o grupo controle (Figura 6D). Porém, os sistemas de discos como o Sof-Lex, apresentam outras limitações, como a dificuldade de acabar e contornar certas superfícies especialmente na região posterior ${ }^{12,15,25}$.

O grupo polido com o sistema PoGo, apresentou maiores médias de rugosidade de superfície para a resina Esthet X, mas sem diferença estatisticamente para o grupo
Sof-Lex (Figura 2). De acordo com St-Georges et al. ${ }^{19}$, o sistema PoGo é composto por partículas micro-diamantadas revestidas, as quais produzem uma superfície similar à obtida com discos de óxido de alumínio. As imagens em microscopia eletrônica de varredura (Figuras 6A e 6B) concordam com esta afirmação.

Para a resina Filtek Supreme XT, não houve diferença entre os grupos antes da escovação (Figura 3), embora a imagem em microscopia eletrônica de varredura do grupo selado com Biscover LV (Figura 8C) apresentasse uma superfície mais uniforme. Os riscos oriundos dos processos de acabamento e polimentos com discos Sof-Lex e Po-Go (Figuras 8A e 8B) não foram suficientes para provocar aumento significativo nos valores de rugosidade de superfície antes da escovação. O sistema Sof-Lex possui alumina como abrasivo, enquanto que o sistema PoGo apresenta partículas de micro-diamantes (pó de diamante micro particulado e óxido de alumínio), podendo causar mais microdefeitos sobre a superfície das restaurações ${ }^{12}$. Segundo $\mathrm{Lu}$ et al. $^{21}$, a diferença de dureza entre os compósitos é muito menor comparada a diferença de dureza entre os sistemas abrasivos de polimento, o que poderia explicar porque estes sistemas afetam mais a rugosidade de superfície do que os compósitos restauradores. Após a escovação, todos os grupos apresentaram aumento na rugosidade de superfície (Ra) à exceção do grupo Biscover LV, o qual não apresentou diferença estatisticamente significante entre os valores de rugosidade antes e após o ensaio mecânico de escovação, tanto para a resina Esthet-X (Figura 4) quanto para a resina Filtek Supreme XT (Figura 5). Com as outras técnicas, o processo de escovação aumentou a rugosidade de superfície. Tais resultados concordam com outros estudos ${ }^{3,8,10}$, mostrando que a aplicação de uma resina fluída seria capaz de aumentar a resistência ao desgaste da resina composta. Pelas imagens em microscopia eletrônica de varredura, observa-se uma menor exposição das partículas dos compósitos após a escovação mecânica nos grupos selados com Biscover LV, tanto para a resina Esthet-X (Figura 7C), quanto para a resina Supreme XT (Figura 9C), comparado aos outros grupos experimentais. A menor exposição das partículas poderia ter contribuído para manutenção dos baixos valores de rugosidade superficial após a escovação.

O uso de resinas fluídas não particuladas para proteger a superfície das restaurações foi sugerido há 20 anos, recomendadas para melhorar suas propriedades e cobrir defeitos e irregularidades formadas devido a remoção de algumas partículas durante $\mathrm{o}$ procedimento de acabamento $^{17}$. O Biscover LV contém, segundo o fabricante, monômeros acrílicos, os quais seriam responsáveis, provavelmente, por manter a superfície dos materiais restauradores com baixo valor de rugosidade de superfície mesmo após a escovação mecânica. Após a escovação, a resina fluída para polimento parece realmente ter cumprido seu papel de selar os defeitos da superfície e mantê-la mais homogênea, uma vez que manteve baixos de rugosidade de superfície. Tais valores situam-se bem abaixo dos limites de rugosidade de superfície estabelecidos por Bollen et al. ${ }^{9}$, segundo o qual valores de rugosidade de superfície acima de $0,2 \mu \mathrm{m}$ facilitariam o acúmulo de placa bacteriana na superfície do compósito.

Vários processos fisiológicos e biológicos podem aumentar a rugosidade de superfície, sendo que, desta forma, estudos precisam determinar qual técnica de acabamento e polimento é mais acessível para cada tipo de resina $^{16}$. O desenvolvimento de métodos de acabamento e 
polimento, que sejam efetivos na manutenção de baixos valores de rugosidade de superfície, sempre será necessário, acompanhando o avanço da ciência tecnológica na formulação de resinas compostas restauradoras cada vez mais diferenciadas.

\section{CONCLUSÃO}

Diferenças significantes na rugosidade de superfície foram observadas entre as resinas compostas e as técnicas de acabamento e polimento avaliadas.

Os maiores valores de rugosidade de superfície (Ra) foram obtidos pela resina Esthet $\mathrm{X}$ devido maior tamanho das partículas de carga expostas depois do polimento.

O ensaio mecânico de escovação causou um aumento na rugosidade para todos os grupos, à exceção do grupo Biscover LV que apresentou os menores valores antes e após a escovação para ambas as resinas.

\section{AGRADECIMENTOS}

Ao apoio financeiro da Fundação de Amparo à Pesquisa de São Paulo - FAPESP (Processos \#2006/50684-0 e \#2006/52400-9).

\section{REFERÊNCIAS}

1. Correr Sobrinho L, Francisco MU, Consani S, Sinhoreti MAC, Consani RLX. Influência da escovação na rugosidade de superfície de materiais restauradores estéticos. Rev Fac Odontol São José dos Campos. 2001; 4(1):47-54.

2. Kobayashi RCO, Consani S, Sinhoreti MAC, Correr Sobrinho L, Góes MF. Rugosidade produzida em restauradores resinosos pela escovação mecânica com escovas dentais complexas. Rev Fac Odontol Univ Passo Fundo. 1998; 3(2):15-24.

3. dos Santos PH, Consani S, Correr Sobrinho L, Coelho Sinhoreti MA. Effect of surface penetrating sealant on roughness of posterior composites resins. Am J Dent. 2003; 16(3):197-201.

4. Baseren M. Surface roughness of nanofill and nanohybrid composite resin and Ormocer-based toothcolored restorative materials after several finishing and polishing procedures. J Biomater Appl. 2004; 19(2):121-34.

5. Yap AU, Yap SH, Teo CK, Ng JJ. Comparison of surface finish of new aesthetic restorative materials. Oper Dent. 2004; 29(1):100-4.

6. Nagem-Filho H, D'Azevedo MT, Nagem HD, Marsola FP. Surface roughness of composite resins after finishing and polishing. Braz Dent J. 2003; 14(1):37-41.

7. Bolanho A, Anauate Netto C, Youssef MN, Do Carmo ARP, Mandelli A. Estudo in vitro da rugosidade de superfície da resinas para dentes posteriores submetidas a diverso tratamentos de superfície. J Bras Dent Estet. 2003;2(5):51-7.

8. Kawai K, Leinfelder KF. Effect of surface-penetrating sealant on composite wear. Dent Mater. 1993; 9(2):108-13.

9. Bollen CM, Lambrechts P, Quirynen M. Comparison of surface roughness of oral hard materials to the threshold surface roughness for bacterial plaque retention: A review of the literature. Dent Mater. 1997; 13(4):258-69.

10. Ratanapridakul K, Leinfelder KF, Thomas J. Effect of finishing on the in vivo wear rate of a posterior composite resin. J Am Dent Assoc. 1989; 118(3):333-5.
11. Lee YK, Lu H, Powers JM. Effect of surface sealant and staining on the fluorescence of resin composites. J Prosthet Dent. 2005;93(3):260-6.

12. Turkun LS, Turkun M. The effect of one-step polishing system on the surface roughness of three esthetic resin composite materials. Oper Dent. 2004;29(2):203-11.

13. Dickinson GL, Leinfelder KF, Mazzer RB, Russell CM. Effect of surface penetrating sealant on wear rate of posterior composite resins. J Am Dent Assoc. 1990; 121(2):251-5.

14. Lee YK, Lu H, Oguri M, Powers JM. Changes in gloss after simulated generalized wear of composite resins. J Prosthet Dent. 2005; 94(4):370-6.

15. Ozgünaltay G, Yazici AR, Görücü J. Effect of finishing and polishing procedures on the surface roughness of new tooth-coloured restoratives. J Oral Rehabil. 2003; 30(2):218-4

16. Gedik R, Hürmüzlü F, Coskun A, Bektas OO, Ozdemir AK. Surface roughness of new microhybrid resin-based composites. J Am Dent Assoc. 2005; 136(8):1106-12.

17. Sarac D, Sarac YS, Kulunk S, Ural C, Kulunk T. The effect of polishing techniques on the surface roughness and color change of composite resins. J Prosthet Dent. 2006; 96(1):33-40.

18. Barbosa SH, Zanata RL, Navarro MF, Nunes OB. Effect of different finishing and polishing techniques surface roughness of microffiled, hybrid and packable composite resins. Braz Dent J. 2005; 16(1):39-44.

19. St-Georges AJ, Bolla M, Fortin D, Muller-Bolla M, Thompson JY, Stamatiades PJ. Surface finish produced on three resin composites by new polishing systems. Oper Dent. 2005; 30(5):593-7.

20. Joniot S, Salomom JP, Dejou J, Grégoire G. Use of two surface analyzers to evaluate the surface roughness of four esthetic restorative materials after polishing. Oper Dent. 2006; 31(1):39-46.

21. Lu H, Roeder LB, Powers JM. Effect of polishing systems on the surface roughness of microhybrid composites. J Esthet Restor Dent. 2003;15(5):297-304.

22. Hunter ML, Addy M, Pickles MJ, Joiner A. The role of toothpastes and toothbrushes in the aetiology of tooth wear. Int Dental J. 2002;52(S5):399-405.

23. McCabe JF, Molyvda S, Rolland SL, Rusby S, Carrick TE. Two-and three-body wear of dental restorative materials. Int Dent J. 2002;52(S5):406-16.

24. Turssi CP, Saad JR, Duarte SL Jr, Rodrigues AL Jr. Composite surfaces after finishing and polishing techniques. Am J Dent. 2000;13(3):136-8.

25. Watanabe $\mathrm{T}$, Miyasaki M, Moore BK. Influence of polishing instruments on the surface texture of resin composites. Quintessence Int. 2006;37(1):61-7.

\section{CONFLITO DE INTERESSES}

Os autores declaram não haver conflitos de interesse.

\section{AUTOR PARA CORRESPONDÊNCIA}

\section{Paulo Henrique dos Santos}

paulosantos@foa.unesp.br

Submetido em 25/09/2017 Aceito em 24/10/2017 